\title{
Mobile health considerations for kidney disease and transplantation
}

\author{
John C. Sieverdes \\ Medical University of South Carolina, College of Nursing, Technology Applications Center for Healthful Lifestyles, Charleston, SC 29425-1600, \\ USA \\ Correspondence to: John C. Sieverdes, PhD. Medical University of South Carolina, College of Nursing, Room SN502, 99 Jonathan Lucas St., \\ Charleston SC 29425-1600, USA. Email: sieverde@musc.edu. \\ Provenance: This is an invited Editorial commissioned by Editor-in-Chief Dr. Steven Tucker, MD (Tucker Medical, Singapore).
}

Received: 16 March 2018; Accepted: 08 May 2018; Published: 18 May 2018.

doi: $10.21037 /$ mhealth.2018.05.02

View this article at: http://dx.doi.org/10.21037/mhealth.2018.05.02

Early on, home-based medical technology products were stand-alone devices used for patient health measurements. These included an array of devices that were modified from their clinic-based counterparts such as blood pressure monitors, glucometers, baby weight scales, and electrocardiogram devices. Programs relied on output metrics viewed on device displays that often required manual recording by the patient. Recorded metrics would then be brought to providers' offices or collected through phone contact. Although these early programs utilized the newest technologies at that time, reporting to providers would be delayed, therefore patients were levied with most of the decisions on how to interpret the data between clinical visits. As technology advanced, devices were equipped with onboard memory to be later downloaded and analyzed. Only when increasing advancements in mobile and internet-based infrastructure penetration made remote real-time monitoring a reality, could data begin to be used for immediate interpretation and action (e.g., blood glucose levels or hypertension levels needing immediate medical response). Real-time data transmission also circumvents non-adherent reporting by the patient while easing their burden. Now, software cut-points and trend analysis can automatically be analyzed to provide immediate direction for patients to take action in addition to simultaneously relaying data to provider groups to signal any further guidance. These technologies along with electronic medical records helped give rise to the discipline of Medical Informatics which is relatively new compared to other sciences. Responsiveness in data analytics will only improve through artificial intelligence and machine learning approaches used in future iterations enabling patients to make more informed decisions while physician offices provide oversight (1).

An important area of increasing importance in the use of mobile health (mHealth) education is in kidney disease patients. These range from chronic kidney disease (CKD), end-stage renal disease (ESRD) requiring renal replacement therapy, to kidney transplant patients. There are a variety of deployed interventions in this population ranging from selfcare education programs, medication adherence programs, deceased and living donor education programs, physical activity and dietary programs, pre-transplant programs, and post-transplant care programs. The purpose of these programs is specific to their outcomes but all enable patients to enact behaviors that improve patient health. There are several barriers that should be considered when interpreting the utility of including kidney disease patients in mHealth programs.

Hardware connectivity still remains a significant barrier although it is improving to increase reach of mHealth interventions. Nationally, $95 \%$ of adults own a cellphone and $77 \%$ are reported to own a smartphone irrespective of race or ethnicity, socioeconomic status, or geographic location (2). The advent of nearly ubiquitous smartphone penetration, even among older adults and lower socioeconomic status, has further improved feasibility to reach increasing number of patients using both smartphone connected or cellular tower connected devices. These include pill trays that automatically report daily or weekly 
medication adherence with time stamped values reported to personal physicians and family members. These may be advantageous for medication management of an array of chronic disease patients through transplant patients' antirejection drug schedules where medication should be taken within a certain time frame each day. Cellular signal maps show expansive coverage regions, though in reality, local geography in urban and rural areas have zones void of cellular or internet connectivity. These barriers, which are unexpected in urban areas, introduce implementation problems for dissemination of mobile health (mHealth) programs.

Another barrier is commonly found in the sustainability of kidney disease mHealth programs. It is evident that the novelty of technology abates soon after it's introduced and is a chronic finding in most studies. Subject interest is fickle in regards to static programs or if only equipping patients to perform data collection without enabling meaningful interpretation of findings (3). It is paramount that methods are examined to address this limitation leading to process and clinically meaningful endpoints. Often, various behavioral theories are required to increase adherence. These may lead to individually tailored programs, or those that alter elements, support, and feedback that are dependent on participant engagement and responses to study protocols or personal goals. Many of the techniques shown to be acceptable during face to face and print material interventions could be translated to interactive mHealth programs. As of late, the ubiquity of technology has allowed many researchers to consider technologybased tools and education using mHealth but technology in of itself is not considered novel as it has been historically treated. Novelty is now more relegated to adapting mHealth methods to specific outcomes or using newly investigated materials and sensors in combination of evidenced-based behavioral programs. One of the often cited mHealth studies for kidney disease patients is the iNephro study which tracks self-managed drug therapy plan and vital signs (4). This app was available on downloadable app stores and was suggestive of high initial uptake, though disuse was evident at 2 months where few users were still using the platform at 1 year. On another note, a review by Whitehead et al. reported on the long-term effectiveness of selfmanagement programs using mobile phone and tablet apps. In the review, 6 of the 9 included studies showed statistically significant improvement in clinical outcomes with only 3 of the 9 showed when restricted to app-based interventions (5). This shows that overall, mHealth programs can be clinically important, but driving adherence may need more attention for sustainability.

The use of multiple devices or separate apps to deliver intervention materials is another barrier in the delivery of mHealth programs for kidney disease patients. An idea to overcome this barrier is the often cited "convergence of technology" as a way to explain the utilization of a singular piece of hardware (e.g., smartphone or smartwatch, etc.) or software [e.g., downloadable app, using existing short-message-system (SMS) texting, etc.] instead of using multiple devices or software applications when developing program materials (6). Centralizing intervention components into one app may increase certain aspects of usability and increase user acceptability by simplifying the system. However, convergence in technology does not necessarily parallel high engagement, sustained use, or effectiveness in mHealth programs. Initial acceptability and sustained use therefore are mutually exclusive and should be investigated separately via longitudinal design. One method to use convergence is to integrate mHealth interventions into an existing app within a smartphone operating system that is already utilized by most users. Unfortunately, the range of manufacturers have produced a fragmented environment with an array of core system apps that are continuously being renewed, created or abandoned making it a difficult proposition.

The largest resource for kidney disease information is through the National Kidney Disease Education Program (NKDEP) which uses web-based education designed to teach patients about kidney disease. The ability to use apps issued by physicians requires the need to address users with low e-literacy, be accessible, confidential, and tailored to the complicated medical care regime of CKD patients (7). Although there are few studies reporting mHealth education practices, a consensus statement from the American Society of Transplantation's Live Donor Community of Practice stated the use of technology should be improved or expanded to better educate kidney disease patients and their support networks (8). Many programs exist to increase patient knowledge about transplantation (9-13). but only a few uses a mHealth approach. In the Living Organ Video Educated Donors program, a tablet-based intervention consisting of interactive video education clips and peer-navigator group video chat groups have shown positive effects $(14,15)$. The other program partnered with Facebook to create an app to share candidate waitlist stories to find a live donor (16). These programs are founded on the social cognitive theory and 
self-determination theory and have found increases in living donor referrals. Other effective programs in increasing knowledge and attitudes on transplantation could be easily adapted for mHealth delivery such as Explore Transplant at Home (17).

Familiarity of smartphone use has risen over the last few years, thus reducing barriers to incorporate smartphone technology in intervention programs aimed at educating and intervening in behavioral change programs. We have found high acceptance of mHealth technology in uncontrolled hypertensive kidney transplant recipients for engagement in their medical regimen with significant improvements in medication intake and sustained blood pressure control evident at 12 months following a 3-month trial $(18,19)$. We also observed high acceptability of a tabletbased video module education and motivation program for Black ESRD patients to learn about the living donor process (20). In other work, kidney transplant waitlist patients were found to be generally acceptable toward mHealth physical activity and dietary improvement programs as long as it is evident that necessary training is covered (21). This was confirmed during a development process of a smartphone-based distance coaching program (22). Importantly, in those studies, as well as others $(23,24)$. a patient/provider-centered iterative design process was used guided by behavioral theories.

mHealth technologies have provided a possible avenue to intervene using behavioral health in the home environment and influence behaviors for sustained engagement in healthy lifestyles, education about transplantation, and medical regimens. Many studies seem to point to adherence being an important topic for study success. In a 2015 review, Hamine and colleagues reported on mHealth chronic disease management on treatment examining adherence and patient outcomes, in their review, 27 out of the 107 articles used randomization, and only 15 out of the 27 showed positive adherence behaviors (25). This shows that adherence outcomes resulting from mHealth control trials have been mixed and although there is potential to facilitate adherence education and self-care tools, the barriers of adherence are notable. Investigation on the underlying behavioral practices between these studies needs to be performed to identify why the successful programs found positive findings compared to the others. The considerations explained here are not confined to kidney disease patients, but expand to other clinical and healthy populations. As researchers, this implies that evaluating and programing for adherence in mHealth studies using behavioral tactics are needed to justifying our methods beyond standard technology usage models.

\section{Acknowledgements}

None.

\section{Footnote}

Conflicts of Interest: The author has no conflicts of interest to declare.

\section{References}

1. Beam AL, Kohane IS. Big Data and Machine Learning in Health Care. JAMA 2018;319:1317-8.

2. Mobile Fact Sheet. Pew Research Center: Internet, Science \& Tech 2017. February 5th, 2018. Available online: http://www.pewinternet.org/fact-sheet/mobile/

3. Boulos MN, Wheeler S, Tavares C, et al. How smartphones are changing the face of mobile and participatory healthcare: an overview, with example from eCAALYX. Biomed Eng Online 2011;10:24.

4. Becker S, Kribben A, Meister S, et al. User profiles of a smartphone application to support drug adherence-experiences from the iNephro project. PLoS One 2013;8:e78547.

5. Whitehead L, Seaton P. The Effectiveness of SelfManagement Mobile Phone and Tablet Apps in Longterm Condition Management: A Systematic Review. J Med Internet Res 2016;18:e97.

6. Bhavnani SP, Narula J, Sengupta PP. Mobile technology and the digitization of healthcare. Eur Heart J 2016;37:1428-38.

7. Diamantidis CJ, Becker S. Health information technology (IT) to improve the care of patients with chronic kidney disease (CKD). BMC Nephrol 2014;15:7.

8. Waterman AD, Morgievich M, Cohen DJ, et al. Living Donor Kidney Transplantation: Improving Education Outside of Transplant Centers about Live Donor Transplantation--Recommendations from a Consensus Conference. Clin J Am Soc Nephrol 2015;10:1659-69.

9. Weng FL, Brown DR, Peipert JD, et al. Protocol of a cluster randomized trial of an educational intervention to increase knowledge of living donor kidney transplant among potential transplant candidates. BMC Nephrol 2013;14:256.

10. Robinson DH, Arriola KR. Strategies to facilitate organ 
donation among African Americans. Clin J Am Soc Nephrol 2015;10:177-9.

11. Rodrigue JR, Cornell DL, Kaplan B, et al. A randomized trial of a home-based educational approach to increase live donor kidney transplantation: effects in blacks and whites. Am J Kidney Dis 2008;51:663-70.

12. Boulware LE, Hill-Briggs F, Kraus ES, et al. Protocol of a randomized controlled trial of culturally sensitive interventions to improve African Americans' and non-African Americans' early, shared, and informed consideration of live kidney transplantation: the Talking About Live Kidney Donation (TALK) Study. BMC Nephrol 2011;12:34.

13. Arriola KR, Robinson DH, Perryman JP, et al. Project ACTS II: organ donation education for African American adults. Ethn Dis 2013;23:230-7.

14. Sieverdes JC, Price M, Ruggiero KJ, et al. Design and approach of the Living Organ Video Educated Donors (LOVED) program to promote living kidney donation in African Americans. Contemp Clin Trials 2017;61:55-62.

15. Sieverdes JC, Nemeth L, Treiber FA, et al. Analysis of a Mobile Health Application to Increase Willingness to Ask for a Living Kidney Donation in Black Renal Patients. 2017 Annual Meeting \& Scientific Sessions; Society of Behavioral Medicine 2017. [In press].

16. Kumar K, King EA, Muzaale AD, et al. A Smartphone App for Increasing Live Organ Donation. Am J Transplant 2016;16:3548-53.

17. Waterman AD, McSorley AM, Peipert JD, et al. Explore Transplant at Home: a randomized control trial of an educational intervention to increase transplant knowledge for Black and White socioeconomically disadvantaged dialysis patients. BMC Nephrol 2015;16:150.

18. McGillicuddy JW, Gregoski MJ, Brunner-Jackson BM, et al. Facilitating medication adherence and eliminating

doi: $10.21037 /$ mhealth.2018.05.02

Cite this article as: Sieverdes JC. Mobile health considerations for kidney disease and transplantation. mHealth 2018;4:13. therapeutic inertia using wireless technology: proof of concept findings with uncontrolled hypertensives and kidney transplant recipients. J Assoc Comp Mach 2012:1-9.

19. McGillicuddy JW, Taber DJ, Mueller M, et al. Sustainability of improvements in medication adherence through a mobile health intervention. Prog Transplant 2015;25:217-23.

20. Sieverdes JC, Treiber FA, Anderson A, et al. Feasibility of the Living Organ Video Educated Donors program (LOVED). 17th Annual State of the Art Winter Symposium: American Society of Transplant Surgeons 2017. [In press].

21. Sieverdes JC, Raynor PA, Armstrong T, et al. Attitudes and perceptions of patients on the kidney transplant waiting list toward mobile health-delivered physical activity programs. Prog Transplant 2015;25:26-34.

22. Sieverdes JC, Bergamin M, Chandler J, et al. Lifestyle Intervention for Transplant Success (LIFTS) Mobile Health Wellness Program for Kidney Wait-Listed Patients: Development and Design. Int Arch Nurs Health Care 2017;3:086.

23. Sieverdes JC, Gregoski M, Patel S, et al. mHealth medication and blood pressure self-management program in Hispanic hypertensives: a proof of concept trial. Smart Homecare Technol Telehealth 2013;1:1-10.

24. Davidson TM, McGillicuddy J, Mueller M, et al. Evaluation of an mHealth Medication Regimen SelfManagement Program for African American and Hispanic Uncontrolled Hypertensives. J Pers Med 2015;5:389-405.

25. Hamine S, Gerth-Guyette E, Faulx D, et al. Impact of mHealth chronic disease management on treatment adherence and patient outcomes: a systematic review. J Med Internet Res 2015;17:e52. 